\title{
Radioiodine - the success story of Nuclear Medicine
}

\section{5th Anniversary of the first use of Iodine-131 in humans}

\author{
V. Ralph McCready ${ }^{1}$
}

Published online: 19 October 2016

(C) Springer-Verlag Berlin Heidelberg 2016

This year marks the 75th anniversary since Saul Hertz first administered radioiodine (I-130-I-131) to a patient on January 1st 1941 in the Massachusetts General Hospital as a treatment for hyperthyroidism. It followed his previous studies on animal thyroid physiology with I-128 to discover how radioiodine could be administered to have a maximal radiation effect in hyperthyroidism. In May 1946, Hertz and Roberts published the follow-up of their series of 29 patients treated with radioactive iodine for hyperthyroidism [1].

Since then, many thousands of patients have been treated successfully with radioiodine for both benign and malignant thyroid disorders. However, the controversies summarized by Mazzaferri in 2004 regarding the best treatment still continue [2].

The use of iodine-131 $\left({ }^{131} \mathrm{I}\right)$, discovered in 1938 by Glenn Seaborg and John Livingood at the University of California, Berkeley, has been the success story in nuclear medicine. It has the advantage of emitting both gamma and beta rays, the former enabling imaging for diagnosis and dosimetry and the latter being valuable for molecular radiotherapy.

The first human studies involved the use of a Geiger counter and, subsequently, scintillation counters to measure the uptake of radioiodine into the thyroid. Distributions within the glands were discovered by plotting the activity in a grid pattern. These were soon superseded by automatic thyroid scanning systems. Rectilinear thyroid scanning was first shown at the International Congress of Radiology in London in 1950 [3]. The rectilinear scans with a focusing collimator

V. Ralph McCready

Ralph.McCready@BSUH.nhs.uk

1 Department Nuclear Medicine Royal Sussex County Hospital East Sussex UK, Brighton, UK demonstrated the different patterns of ${ }^{131}$ I uptake in normal, hyperactive and cancerous thyroid tissue discovering the detail of iodine physiology. The advent of tomographic imaging with camera systems has enabled the activity to be imaged in 3D making improved dosimetry possible.

Even in the early days it was clear that I-131 for thyroid cancer therapy was effective at a time when the onset of most cancers was a death sentence. One of the first patients who presented at the Royal Cancer Hospital (London) in 1949 had a mainly inoperable differentiated thyroid cancer and metastases requiring a tracheotomy responded dramatically after three administrations $(50,88,100,230 \mathrm{mCi})$ of radioiodine [4]. She was alive and well with grandchildren 24 years later. In New York City, Seidlin had demonstrated that following thyroid ablation, reducing the thyroid's competition for radioiodine, metastases would show uptake [5]. Since then, many papers have recorded the prolonged survival achieved following radioiodine therapy. The overall survival rate in patients with differentiated carcinoma in general is about $85 \%[6]$.

The treatment over the years varied little until 2012 following the publication of the HiLo trial [7]. It would seem that the initial choice of $100 \mathrm{mCi}(3.7 \mathrm{GBq})$ for post-surgical ablation centered on a round figure rather than a calculated prescription. The use of $30 \mathrm{mCi}(1.1 \mathrm{GBq})$ was based on the desire to avoid hospitalization rather than science. However, it can be repeated until there is no evidence of residual normal thyroid tissue [8]. This would not seem to be a problem in terms of disease outcome. Up to $27 \%$ of patients can be ablated by one activity, although this figure must depend upon the volume of normal thyroid tissue remaining. If the administered activity for ablation is chosen without reference to dosimetry, then the amount should balance minimizing the risk of inducing a secondary primary cancer against the aim of decreasing the 
chance of recurrence. In the HiLo UK multicentre study where thyroid surgery was performed in specialist centres, $30 \mathrm{mCi}$ $(1.1 \mathrm{GBq})$ resulted in $49 \%$ ablation with a single administered activity while $51 \%$ required further activity. With a median follow-up of 24 years (range 4-34 years), those requiring higher activity had a reduced disease-free survival of $81 \%$ compared with $95 \%$ for those ablated with the lower activity [9]. Although the difference in survival is small, this raises the possibility that the lower activity does not eliminate occult residual primary or secondary disease. It also raises the question whether lower administered activities should be used to reduce the frequency of second primary malignancies (SPMs). The paper by Iyer et al. has shown a steady rise of SPM over the years [10]. However, another large study of 1943 patients with differentiated thyroid cancer found that with a follow up of 9 years, $9 \%$ of radioactive iodine (RAI)-treated patients were diagnosed with SPMs while there were $10.5 \%$ SPMs in non-RAI-treated patients [11].

Once recurrence from a differentiated thyroid carcinoma occurs, the outlook is poor unless the recurrence can be ablated with repeated high activities of RAI. In a paper by Durante et al. reporting a series of 444 patients, negative imaging studies were achieved in $43 \%$ while those who didn't had a 10year survival of $19 \%$ [12]. These results pose the question of whether occult disease was not ablated at the time of ablation and if the progress of the disease is complicated by dedifferentiation of all or part of the tumour. Thus, there is an argument for administering an initial high tumour ablative dose [the original $100 \mathrm{mCi}(3.7 \mathrm{GBq})$ ] rather than limiting the aim to the ablation of normal residual tissue. On the other hand, a new clinical trial is advocating omitting the ablation activity in a group of patients with known favourable characteristics[13]. This can be a difficult decision for patients who are familiar with the successful outcome of those receiving higher activities.

The advent of 3D imaging and dosimetry has made it possible to aim for a specific absorbed dose rather than the nonscientific approach of using arbitrarily fixed activities. Progress is being made in determining the absorbed dose necessary to ablate normal tissue and differentiated thyroid cancer. Flux et al. demonstrated a wide variation in the maximum voxel dose to thyroid remnants from 7 to 570 Gy in patients who had a fixed administered activity of $3.0 \mathrm{GBq}$. The minimum absorbed dose for successful ablation was 49 Gy [14]. The study by Wierts et al. showed that the absorbed dose threshold for maximizing complete response prediction was $90 \mathrm{~Gy}$ for normal remnants and $40 \mathrm{~Gy}$ for metastases [15]. For bone metastases, the required ablative absorbed dose seems to be much higher, being over 600 Gy for an $80 \%$ response [16]. The wide range of absorbed doses may reflect the nonuniformity of dose distribution. To change the approach away from using fixed activities it will be necessary to use ${ }^{124} \mathrm{I}$ positron emission tomography/computed tomography (PET/
CT) for accurate dosimetry and to determine the ablative absorbed dose for normal remnants. Alternatively, the approach could be to determine the bone marrow absorbed dose with a constraint of $<2$ Gy and use it to calculate the maximum safe administered activity to minimize the possibility of recurrent disease from occult primary or secondary cancer.

In the case of pediatric differentiated thyroid carcinoma, the situation is complicated by the expectation of a longer potential disease-free period increasing the risk of second primary malignancies. The use of fixed activities calculated using age or weight quite rightly is being replaced by a more scientific dosimetric approach. This will take time to become established. In the meantime, one approach is to personalize the prescription by adjusting the ablation-administered activity using body weight, surface area and fractional uptake in the thyroid remnant [17].

\section{Benign thyroid disease}

Hertz's original use of radioiodine was in a patient with Grave's disease. 75 years later, Wang and Qin have concluded on the basis of a meta analysis that 'Radioiodine therapy is associated with a higher hyperthyroid cure rate and lower relapse rate' than anti-thyroid drugs and they 'would recommend radioiodine therapy as the treatment of choice for patients with Graves' disease' [18]. The issues with such treatment are the side-effects of radioiodine, hypothyroidism and the potential carcinogenic effects of radiation. The latest USA guidelines suggest that the aim should be total ablation with replacement thyroid hormone therapy [19]. However, many patients would prefer to be euthyroid without medication for life. The European Association of Nuclear Medicine (EANM) guidelines are more ambivalent. According to the EANM guidelines, in patients with hyperthyroidism, the aim of treatment with ${ }^{131} \mathrm{I}$ is to achieve a non-hyperthyroid status, which can be euthyroid or hypothyroid, recompensated by T4 medication [20]. Permanent hypothyroidism can be achieved relatively easily by administering a high activity to deliver an ablative dose. The American Thyroid Association recommends a fixed activity of $10-15 \mathrm{mCi}(370-555 \mathrm{MBq})$. A $15 \mathrm{mCi}(555 \mathrm{MBq})$ administration results in an $81 \%$ success rate [19]. The EANM guidelines indicate that complete ablation requires an absorbed dose of 200-300 Gy [20].

Various methods of calculating the required activity to ablate the thyroid have been proposed. The data required include the mass of the thyroid gland, the target dose and the radioiodine uptake and retention. This requires time from both the scientist and the patient. However, more research is still required to define the optimum absorbed dose and the administered activity to achieve it. The research should also investigate what factors influence the absorbed dose/administered activity relationship. It may be that in spite of the 
complications, an intravenous administration could minimize at least one of the factors which contribute to the range of absorbed doses seen clinically.

Patients with hyperactivity due to autonomous functioning nodules have traditionally been given high activities. The long-term outcome from this approach must depend upon the relative uptake between the nodule(s) and the normal thyroid tissue. In patients where a target absorbed dose was determined, a success rate of up to $100 \%$ has been achieved. The incidence of subsequent hypothyroidism was between 10 and $20 \%$ [21]. In another study, there was a $30 \%$ incidence of hypothyroidism at the end of the year following treatment using 370-740 MBq I-131 [22]. Hypothyroidism could be reduced if the normal tissue was protected by the direct injection of the radioiodine into the nodule under ultrasound control [23]. A similar anatomical approach using radiofrequency ablation is already being used with success $[24,25]$.

\section{Conclusion}

Radioiodine for the treatment of thyroid disorders has been the success story of nuclear medicine. It involves the use of an economic readily available radionuclide which is highly concentrated by the thyroid gland. The results of its use in the treatment of differentiated cancer of the thyroid are excellent, acting as a model for molecular radiotherapy. In spite of being in clinical use for 75 years, there are still areas for research which could improve the outcomes in benign and malignant thyroid disease. The idea of using fixed activities and activities calculated based on body weight seem illogical and are based on the experience of drug therapy where the drug diffuses throughout the body. In contrast, radiopharmaceuticals target and concentrate in pathologies and organs and it is the absorbed dose from the tissue uptake that is important. In external beam radiation therapy, the major effort is in delivering radiation in the most effective manner and minimizing side-effects by using the appropriate dose and a treatment plan. Perhaps it is time to review our approach to molecular radiotherapy, especially for thyroid disease where we have a radionuclide with high concentration and the means of measuring its physiology in real time and with high resolution.

\section{References}

1. Hertz S, Roberts A. Radioactive iodine in the study of thyroid physiology; the use of radioactive iodine therapy in hyperthyroidism. J Am Med Assoc. 1946;131:81-6.

2. Mazzaferri E. A randomized trial of remnant ablation-in search of an impossible dream? J Clin Endocrinol Metab. 2004;89(8):3662-4.
3. Webb S. From the watching of shadows: the origins of radiological tomography. 1990.: Bristol New York: Adam Hilgey. 1990.

4. Smithers DW. Some varied applications of radioactive isotopes to the localization and treatment of tumors. Acta Radiol. 1951;35(1): 49-61.

5. Siegel E. The beginnings of radioiodine therapy of metastatic thyroid carcinoma: a memoir of Samuel M. Seidlin, M. D. (1895$1955)$ and his celebrated patient. Cancer Biother Radiopharm. 1999;14(2):71-9.

6. Luster M, Clarke SE, Dietlein M, Lassmann M, Lind P, Oyen WJ, et al. Guidelines for radioiodine therapy of differentiated thyroid cancer. Eur J Nucl Med Mol Imaging. 2008;35(10):1941-59.

7. Mallick U, Harmer C, Yap B, Wadsley J, Clarke S, Moss L, et al. Ablation with low-dose radioiodine and thyrotropin alfa in thyroid cancer. N Engl J Med. 2012;366(18):1674-85.

8. Comtois R, Theriault C, Del VP. Assessment of the efficacy of iodine-131 for thyroid ablation. J Nucl Med. 1993;34(11):1927-30.

9. Welsh L, Powell C, Pratt B, Harrington K, Nutting C, Harmer C, et al. Long-term outcomes following low-dose radioiodide ablation for differentiated thyroid cancer. J Clin Endocrinol Metab. 2013;98(5):1819-25.

10. Iyer NG, Morris LG, Tuttle RM, Shaha AR, Ganly I. Rising incidence of second cancers in patients with low-risk (T1N0) thyroid cancer who receive radioactive iodine therapy. Cancer. 2011;117(19):4439-46.

11. Hirsch D, Shohat T, Gorshtein A, Robenshtok E, Shimon I, Benbassat C. Incidence of Nonthyroidal Primary Malignancy and the Association with (131)I Treatment in Patients with Differentiated Thyroid Cancer. Thyroid. 2016;26(8):1110-6.

12. Durante C, Haddy N, Baudin E, Leboulleux S, Hartl D, Travagli JP, et al. Long-term outcome of 444 patients with distant metastases from papillary and follicular thyroid carcinoma: benefits and limits of radioiodine therapy. J Clin Endocrinol Metab. 2006;91(8):2892-9.

13. Mallick U, Harmer C, Hackshaw A, Moss L. Iodine or Not (IoN) for low-risk differentiated thyroid cancer: the next UK National Cancer Research Network randomised trial following HiLo. Clin Oncol (R Coll Radiol). 2012;24(3):159-61.

14. Flux GD, Haq M, Chittenden SJ, Buckley S, Hindorf C, Newbold K, et al. A dose-effect correlation for radioiodine ablation in differentiated thyroid cancer. Eur J Nucl Med Mol Imaging. 2010;37(2):270-5.

15. Wierts R, Brans B, Havekes B, Kemerink GJ, Halders SG, Schaper NN, et al. Dose-response Relationship in Differentiated Thyroid Cancer Patients Undergoing Radioiodine Treatment Assessed by Means of 124I PET/ CT. J Nucl Med. 2016;57(7):1027-32.

16. Jentzen W, Verschure F, van ZA, van de KR, Wierts R, Schmitz J, et al. Response Assessment of Bone Metastases from Differentiated Thyroid Cancer Patients in the Initial Radioiodine Treatment Using Iodine-124 PET Imaging. J Nucl Med. 2016.

17. Franzius C, Dietlein M, Biermann M, Fruhwald M, Linden T, Bucsky P, et al. Procedure guideline for radioiodine therapy and 131iodine whole-body scintigraphy in paediatric patients with differentiated thyroid cancer. Nuklearmedizin. 2007;46(5):224-31.

18. Wang J, Qin L. Radioiodine therapy versus antithyroid drugs in Graves' disease: a meta-analysis of randomized controlled trials. Br J Radiol. 2016:20160418.

19. Ross DS, Burch HB, Cooper DS, Greenlee MC, Laurberg P, Maia AL, et al. 2016 American Thyroid Association Guidelines for Diagnosis and Management of Hyperthyroidism and other causes of Thyrotoxicosis. Thyroid. 2016.

20. Stokkel MP, Handkiewicz JD, Lassmann M, Dietlein M, Luster M. EANM procedure guidelines for therapy of benign thyroid disease. Eur J Nucl Med Mol Imaging. 2010;37(11):2218-28. 
21. Reiners C, Schneider P. Radioiodine therapy of thyroid autonomy. Eur J Nucl Med Mol Imaging. 2002;29 Suppl 2:S471-S8.

22. Aktas GE, Turoglu HT, Erdil TY, Inanir S, Dede F. LongTerm Results of Fixed High-Dose I-131 Treatment for Toxic Nodular Goiter: Higher Euthyroidism Rates in Geriatric Patients. Mol Imaging Radionucl Ther. 2015;24(3):94-9.

23. McCready VR, Dizdarevic S, Aplin M. Interventional nuclear medicine. Eur J Nucl Med Mol Imaging. 2013;40(7):1044-6.
24. Happel C, Korkusuz H, Koch DA, Grunwald F, Kranert WT. Combination of ultrasound guided percutaneous microwave ablation and radioiodine therapy in benign thyroid diseases. A suitable method to reduce the 131I activity and hospitalization time? Nuklearmedizin. 2015;54(3):118-24.

25. Sung JY, Baek JH, Jung SL, Kim JH, Kim KS, Lee D, et al. Radiofrequency ablation for autonomously functioning thyroid nodules: a multicenter study. Thyroid. 2015;25(1):112-7. 